\title{
Chapter 21 \\ Complement in Action: An Analysis of Patent Trends from 1976 Through 2011
}

\author{
Kun Yang, Robert A. DeAngelis, Janet E. Reed, Daniel Ricklin, and John D. Lambris
}

\begin{abstract}
Complement is an essential part of the innate immune response. It interacts with diverse endogenous pathways and contributes to the maintenance of homeostasis, the modulation of adaptive immune responses, and the development of various pathologies. The potential usefulness, in both research and clinical settings, of compounds that detect or modulate complement activity has resulted in thousands of publications on complement-related innovations in fields such as drug discovery, disease diagnosis and treatment, and immunoassays, among others. This study highlights the distribution and publication trends of patents related to the complement system that were granted by the United States Patent and Trademark Office from 1976 to the present day. A comparison to complementrelated documents published by the World Intellectual Property Organization is also included. Statistical analyses revealed increasing diversity in complement-related research interests over time. More than half of the patents were found to focus on the discovery of inhibitors; interest in various inhibitor classes exhibited a remarkable transformation from chemical compounds early on to proteins and antibodies in more recent years. Among clinical applications, complement proteins and their modulators have been extensively patented for the diagnosis and treatment of eye diseases (especially agerelated macular degeneration), graft rejection, cancer, sepsis, and a variety of other inflammatory and immune diseases. All of the patents discussed in this chapter, as well as those from other databases, are available from our newly constructed complement patent database: www.innateimmunity.us/patent.
\end{abstract}

\subsection{Introduction}

\subsubsection{Complement: A Powerful Network for Immune Surveillance}

Complement has traditionally been viewed as a major early defense system to protect the host from pathogenic organisms, and it is known to be intimately involved in regulation of immune responses. The three major pathways of complement activation are the classical, lectin, and alternative pathways (Fig. 21.1), though in certain circumstances, complement can be activated downstream of these

K. Yang • R.A. DeAngelis • D. Ricklin • J.D. Lambris $(\varangle)$

Department of Pathology and Laboratory Medicine, University of Pennsylvania, 401A Stellar-Chance Building,

422 Curie Blvd, Philadelphia, PA 19104, USA

e-mail: lambris@upenn.edu

J.E. Reed

Potter Anderson \& Corroon LLP, Wilmington, DE, USA 


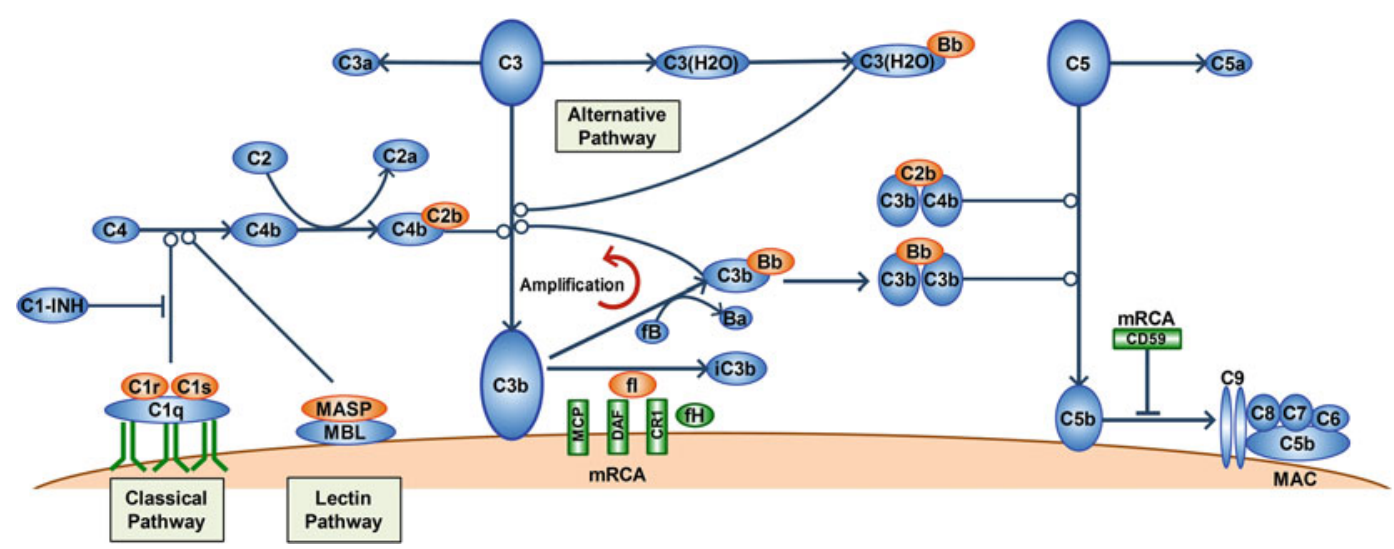

Fig. 21.1 Activation and regulation of the complement system. Complement activation has traditionally been described as occurring through three initiation pathways (classical, lectin, and alternative). External proteases are also known to activate complement, independent of these pathways (at the level of C3 and C5), but for simplicity, they are not shown here. The classical and lectin pathways share common mechanisms and differ mainly in their initial activating components. The classical pathway is initiated by the binding of $\mathrm{C} 1 \mathrm{q}$ to antigen-antibody complexes, while the lectin pathway is triggered by the binding of MBL or ficolins to pathogen surfaces. This binding leads in both cases to the cleavage of $\mathrm{C} 2$ and $\mathrm{C} 4$ to form the $\mathrm{C} 4 \mathrm{~b} 2 \mathrm{~b}$ complex ( $\mathrm{C} 2 \mathrm{~b}$ refers to the protease fragment of $\mathrm{C} 2$, which has also been referred to as $\mathrm{C} 2 \mathrm{a}$ in previous literature), though this activity can be blocked by the $\mathrm{C} 1$ inhibitor $(\mathrm{C} 1-\mathrm{INH})$; $\mathrm{C} 4 \mathrm{~b} 2 \mathrm{~b}$ then promotes the cleavage of the central complement component $\mathrm{C} 3$ and generates both the anaphylatoxin $\mathrm{C} 3 \mathrm{a}$ and the opsonin $\mathrm{C} 3 \mathrm{~b}$. The production of $\mathrm{C} 3 \mathrm{~b}$ can also be promoted by the interaction of $\mathrm{C} 3\left(\mathrm{H}_{2} \mathrm{O}\right)$ with factor $\mathrm{B}(f B)$ and factor $\mathrm{D}(f D)$ to form an initial alternative pathway $\mathrm{C} 3$ convertase. $\mathrm{C} 3 \mathrm{~b}$ then combines with $\mathrm{fB}$ and $\mathrm{fD}$ to form the major alternative pathway $\mathrm{C} 3$ convertase $(C 3 b B b)$, which leads to an amplification loop for further cleavage of $\mathrm{C} 3$. The combination of C3b with the $\mathrm{C} 4 \mathrm{~b} 2 \mathrm{~b}$ or $\mathrm{C} 3 \mathrm{bBb}$ complexes leads to the formation of $\mathrm{C} 5$ convertases, which cleave $\mathrm{C} 5$ into the $\mathrm{C} 5 \mathrm{a}$ anaphylatoxin and $\mathrm{C} 5 \mathrm{~b}$. C5b then binds to $\mathrm{C} 6, \mathrm{C} 7, \mathrm{C} 8$, and $\mathrm{C} 9$ to form the membrane attack complex (MAC). To control the excessive activation of complement, soluble $\mathrm{fH}$ and membrane-associated regulators of complement activity ( $m R C A s)$ such as CR1, DAF, and MCP act as factor I ( $I$ I) cofactors and degrade C3b to iC3b. CD59, on the other hand, inhibits the assembly of the MAC by binding to $\mathrm{C} 8$ and/or C 9

initiation events through the actions of external proteases. Though various factors can initiate complement activation, the three pathways all converge at the cleavage of $\mathrm{C} 3$ by $\mathrm{C} 3$ convertases, resulting in the production of $\mathrm{C} 3 \mathrm{a}$ and $\mathrm{C} 3 \mathrm{~b} . \mathrm{C} 3 \mathrm{a}$ is an anaphylatoxin with chemoattractant properties and cell-signaling abilities through the $\mathrm{C} 3$ a receptor, while $\mathrm{C} 3 \mathrm{~b}$ and its cleavage product $\mathrm{iC} 3 \mathrm{~b}$ can act as opsonins, being deposited on pathogenic cells and targeting them for phagocytosis or clearance, as well as the activation of immune cells through an interaction with complement receptors (Markiewski and Lambris 2007). C3b also initiates a self-amplification loop for further complement activation, as its deposition on cells is the basis for the formation of alternative pathway C3 convertase complexes, as well as C5 convertases that cleave $\mathrm{C} 5$ to continue downstream activation of the cascade. The cleavage of $\mathrm{C} 5$ produces the powerful anaphylatoxin $\mathrm{C} 5 \mathrm{a}$; like $\mathrm{C} 3 \mathrm{a}$, C5a (though signaling via the $\mathrm{C} 5 \mathrm{a}$ receptor [C5aR/CD88]) can contribute to immune cell accumulation and activation, generally promoting inflammatory responses. $\mathrm{C} 5 \mathrm{~b}$, which is also produced by the cleavage of $\mathrm{C} 5$, triggers the formation of a downstream membrane attack complex (MAC); this terminal component of complement activation can be involved in signaling at sublytic doses or can function to kill certain strains of bacteria through insertion in the membrane and subsequent cell lysis (Ricklin et al. 2010).

\subsubsection{Complement in Infection and Disease}

Because of the essential role of complement in the defense of the host against intruders, decreased activation of the complement system or deficiency in complement components can increase the risk 
of infection and cause various pathological conditions (Skattum et al. 2011). For example, alcoholic cirrhosis patients with low serum C3 concentrations and decreased hemolytic complement activity have been reported to have an increased risk of infections (Homann et al. 1997). Deficiencies of components of the classical pathway such as $\mathrm{C} 1 \mathrm{q}, \mathrm{C} 2$, and $\mathrm{C} 4$ have been found to be strongly associated with systemic lupus erythematosus (SLE) (Pickering et al. 2000; Pettigrew et al. 2009). On the other hand, uncontrolled, inappropriate, or excessive complement activity can cause damage to host cells and give rise to many diseases ranging from autoimmune to inflammatory pathologies (Markiewski and Lambris 2007; Holers 2003; Ricklin and Lambris 2007; Lambris and Sahu 2001). Indeed, the dual role of complement is illustrated by several pathological conditions. For example, the early increase of complement activation during sepsis may relate to the beneficial opsonization of bacteria. However, complement activation during subsequent phases of sepsis can amplify the initial insult, leading to inflammatory activity, tissue injury, and finally to multiple organ failure and death in many cases (Markiewski et al. 2008a).

Although complement has traditionally been seen as a defense mechanism against pathogens, it has been shown in recent years to also play a role in general immune surveillance, as well as a host of other immune-related functions and inflammatory diseases. Tissue regeneration, lipid metabolism, transplant rejection, age-related macular degeneration (AMD), rheumatoid arthritis, hemodialysisassociated thrombosis, and cancer are just a few of the increasing number of physiological and pathological processes in which complement activity has been implicated (Ricklin et al. 2010). As with pathogen defense, complement can act as both a protective and damaging factor in many of these conditions. For example, complement inhibits tumor growth through antibody-dependent cell-mediated cytotoxicity (ADCC) and complement-dependent cytotoxicity (CDC) (Markiewski and Lambris 2009). However, it has been found that tumors express high amounts of membrane-associated regulators of complement activity (mRCAs) and secrete fluid-phase regulators, which inhibit the activation of complement and contribute to tumor growth (Markiewski and Lambris 2009). In addition, a recent study has shown that complement can promote the progression of tumors in a mouse model of cervical cancer through the generation of C5a in the tumor microenvironment, which enhances the activity of immune-suppressing cells (Markiewski et al. 2008b). Thus, complement activity is critical for certain homeostatic or immune processes, but in some instances, such as over-activation or improper timing or length of activity, it can become detrimental to the overall health of the host.

\subsubsection{Protecting Intellectual Property in Complement Research: An Analysis}

The multifaceted role of complement in immune defense and disease makes it an attractive biomarker for diagnostic purposes and an obvious target for intervention by complement therapeutics. Thus, it is not surprising to see research and development in complement diagnostics and therapeutics reflected in a large number of granted patents and an increasing number of new patent applications being filed every year. Here, we investigate and analyze the patent landscape as it applies to complement, focusing on granted patents and applications as published by the United States Patent and Trademark Office (USPTO) and, in part, the World Intellectual Property Organization (WIPO). We highlight the history and emerging trends in patent publications on the complement system in different fields. We performed statistical analyses on (1) the specific complement components being targeted by innovations, (2) the areas in which complement-related patents have been focused, (3) the different complement inhibitor forms developed for experimental and potential therapeutic use, and (4) the clinical areas upon which relevant patents were centered. To further assist researchers in the complement field, a complement patent database has been constructed, and all the documents used in the statistical analyses presented here (from the USPTO and WIPO), as well as those retrieved from the European Patent Office (EPO) and Japanese Patent Office (JPO), are available from the database website: 
www.innateimmunity.us/patent. Patents stored in the database have full-text links and are classified in terms of target, disease, application, and entity type. An advanced search function is available for finding patents based on specific interests. This database was intended to be thorough; however, there is no way to ensure that all complement-related patent publications are included. Accordingly, this database and the analysis of it as described in this chapter should be taken as reflecting trends only; they are not intended to replace a firsthand search of the USPTO and WIPO databases.

\subsection{Study Design and Methodology}

\subsubsection{Terminology}

It should be noted that in the text and figures here, the term "documents" is used to refer to all records retrieved from a database (both applications and granted patents). When a distinction is required between "applications" and "granted patents," these specific terms are used (with "US-granted patents" or "US applications" referring to those obtained from the USPTO). The term "publications" is used in a general sense to refer to data currently being discussed (in most cases, US-granted patents).

\subsubsection{Data Retrieval and Analysis}

To identify complement-related patent data as presented in this study, the databases of the USPTO and the WIPO were searched, retrieving all documents (applications and granted patents) whose title or abstract contained the keyword "complement." It should be noted that the WIPO searches were confined to publications that were part of the Patent Cooperation Treaty (PCT), which at the time consisted of 144 states. Dates of coverage for the searches were from the earliest dates for which data was available through December 31, 2011. For the USPTO database, the earliest date for records was January 1, 1976, for granted patents and March 15, 2001, for applications (no application records were available prior to this date), while for the WIPO/PCT, which did not distinguish between applications and granted patents, records started on January 1, 1978.

Once all complement-related documents were retrieved, the Python programming language was used to organize and process the resulting enormous amount of data. Documents that were not related to the complement system, as determined by using rules to exclude those containing keywords unrelated to biological systems (e.g., "automobile," "logic calculator," etc.), were discarded from the data pool; the remaining documents were individually examined to verify their association with the complement system.

Although both the USPTO and WIPO/PCT databases were searched, in most cases, only the USPTO-granted patents were used for the comprehensive data analysis because of the difficulty in ensuring that all duplications could be eliminated between these two databases and between US applications and granted patents, which would otherwise skew results when trends were being examined. Also, the USPTO database contained older data, and it was found that in most cases, inventors wanted to protect their intellectual property in the USA as well as abroad, and thus they filed for patents from both offices. Therefore, the USPTO results, specifically the granted patents, were seen to adequately represent general patent trends. However, a comparison between US patents and applications, as well as the US and WIPO/PCT database results, is presented in Fig. 21.2.

Once patents were verified as relating to the complement system, they were classified into various groups based on target, application, or disease, as presented in the results below. 

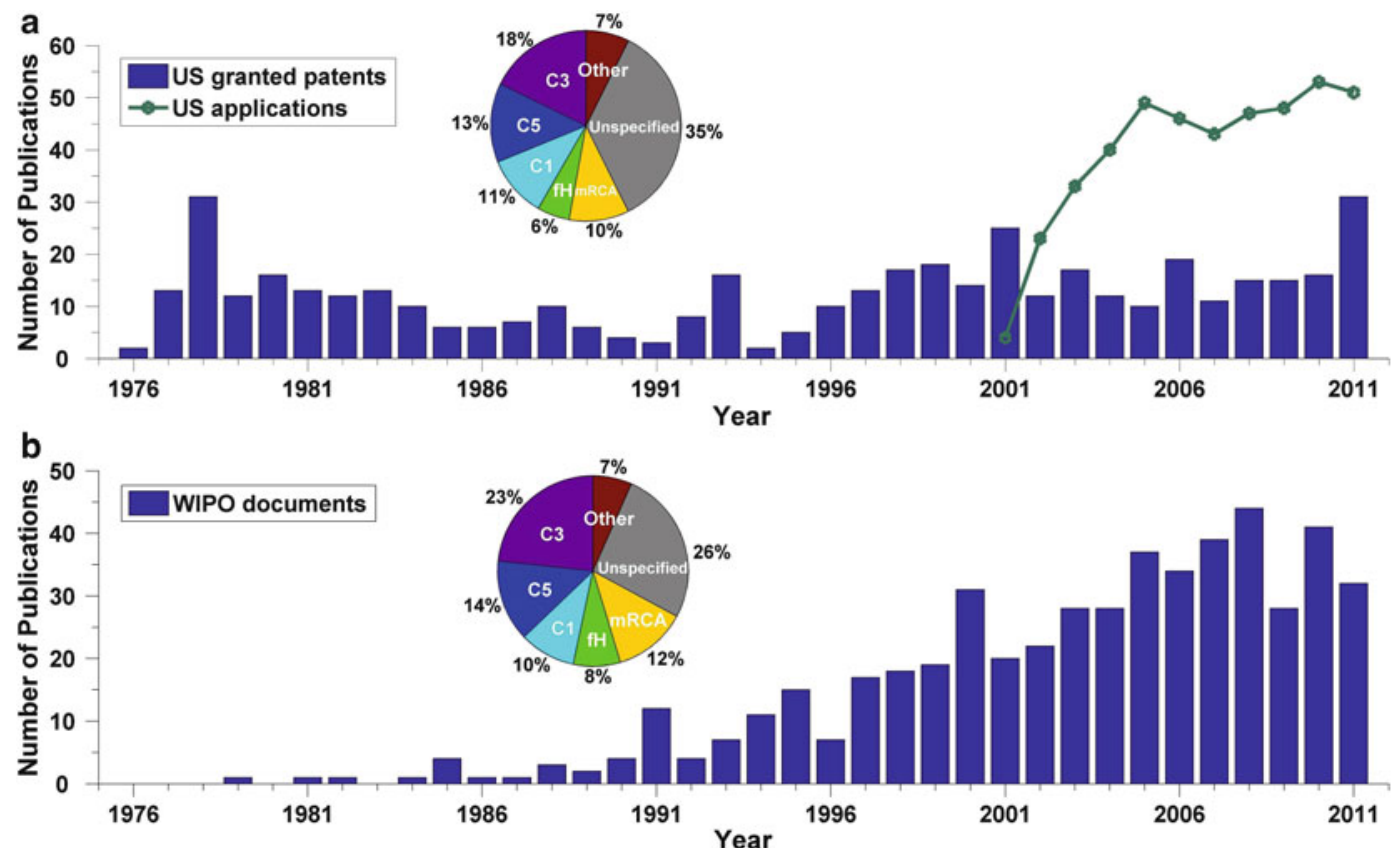

Fig. 21.2 General publication trend for documents targeting complement components. Granted patents and applications involving the complement system were collected, covering all available US patents and US applications (a) and WIPO/ PCT documents (b) from 1976 to the end of 2011. Yearly trends for all complement-related publications are shown as column or line graphs. Publications were also classified by their complement targets, and the distribution was analyzed and displayed as a pie chart showing the top five targets $(C 1, C 3, C 5, f H$, and $m R C A s)$ from each database; the remaining complement components are grouped under "Other." The "Unspecified" category refers to publications that did not specify a specific complement target. For the analysis displayed in the pie chart in (a), duplications between US-granted patents and applications (for the years 2001 onward), in which two or more documents were found to have the same title, abstract, and claims, were removed for accuracy (only one document was included in the analysis). Most likely, these duplicates would represent an application and the resulting granted patent(s) and thus refer to the same innovation. However, if two or more documents were found to have the same title and abstract but differences in their claims, then these were considered two separate documents (as they could represent continuations-in-part), and both were included in the data shown

\subsubsection{Patent Database}

The complement patent database was created using MySQL. The web interface was implemented using PHP language. The database is running on a Windows 2003 server with IIS6 as the http server. To ensure comprehensive coverage, results were included from not only the USPTO and WIPO/PCT but also the EPO and JPO.

\subsection{Results and Discussion}

\subsubsection{Patent Trends Reflect an Increased Interest in Complement Research}

More than 1,000 patent documents related to the complement system have been published by the USPTO and WIPO/PCT since 1976. The publications have followed a general trend of yearly growth 
(Fig. 21.2a, b, graphs). Specifically, the number of US applications has increased rapidly over the last 10 years, averaging about 48 per year since 2005 (Fig. 21.2a, graph). Compared to US applications, the number of US-granted patents has remained steadier. When analyzed by decade, the average numbers of granted patents in the 1980s, 1990s, and 2000s were 10, 10, and 16, respectively. Interestingly, over the last decade, the only year for which application data are available, the number of granted patents was less than half the number of applications. As is true for the trend in US applications, the number of documents issued by the WIPO/PCT has generally increased over the years (Fig. 21.2b, graph). The distribution patterns of US documents (applications and granted patents) and WIPO documents targeting different complement components were quite similar (Fig. 21.2a, b, pie charts), with $\mathrm{C} 3, \mathrm{C} 5, \mathrm{C} 1$, factor $\mathrm{H}$ (fH), and mRCAs (defined in this study as including MCP/CD46, DAF/CD55, CR1/CD35, and CD59) as the top five targets. Documents targeting these five complement components accounted for more than half of all publications. Correspondingly, the number of documents targeting other complement components was quite low, consisting of only $7 \%$ of all publications. Taken together, the data indicate that interest in the complement system has grown dramatically since the 1970s, with a particularly striking increase over the last 20 years. This is not entirely surprising, since there has been a corresponding increase in knowledge about the complement system during this same time period. Also, the perception of complement has shifted from that of an innate immune system that is primarily important for host defense against pathogens to a much more complex and cross-interactive pathway of proteins involved in a multitude of pathological and homeostatic responses (Ricklin et al. 2010). Thus, interest in and innovations related to complement have grown along with this gain in knowledge. It is also worth noting that while the number of complementrelated US-granted patents has remained relatively steady during the past two decades, there has been a blossoming of international publications. This growth may reflect not only an increasing presence of complement research outside of the USA but also the general globalization trends leading US researchers to seek patents both at home and abroad; when the number of applications is taken into account, it is obvious that complement-related research remains prolific in the USA.

\subsubsection{Target Analysis Reveals a Shift in the Focus of Complement Patents}

We performed further analysis only on US-granted patents (see Methodology for the rationale behind excluding US applications and WIPO/PCT documents) in order to ascertain publication trends in the application of complement components and their modulators to various fields. As with the analysis of all USPTO and WIPO/PCT documents, C3, C5, mRCAs, C1, and fH were found to be the five most popular targets for US-granted patents, with an overall trend for yearly growth since the early $1980 \mathrm{~s}$ (Fig. 21.3, graphs). It should be noted though that most patents granted in the 1970s and 1980s did not specify their targets. Instead, a large majority of those patents concerned the development of general chemical inhibitors of the complement system, for which the mechanism of action was likely not known, although several also involved the development of assays to detect complement activity. Before 1990, C1 was the most popular of the specified targets, with few patents relating to $\mathrm{fH}$ and mRCAs, the only other complement components specifically targeted during this time period (Fig. 21.3, pie charts). C1 was an early popular target for various types of inhibitors. Specific patents were published for amidine compounds with a $\mathrm{C} 1$ esterase inhibitory action useful for treating pancreatitis, hemorrhagic diseases, and thrombosis (Fujii et al. 1986, 1987). C1 was also utilized in detection assays, including one to reveal rheumatoid factors in blood (O'connell et al. 1987; Hallgren and Wide 1980; Taguchi et al. 1991), and another early patent described a method for its purification using chromatography (Bing 1983). From 1990 onward, as knowledge about the complement system grew, research interests became more diverse, not only in terms of initiators of complement activity, but also those components involved in its amplification, regulation, and downstream signaling. C3 replaced $\mathrm{C} 1$ as the dominant factor of interest and was the subject of $19 \%$ of all publications, while interest in 


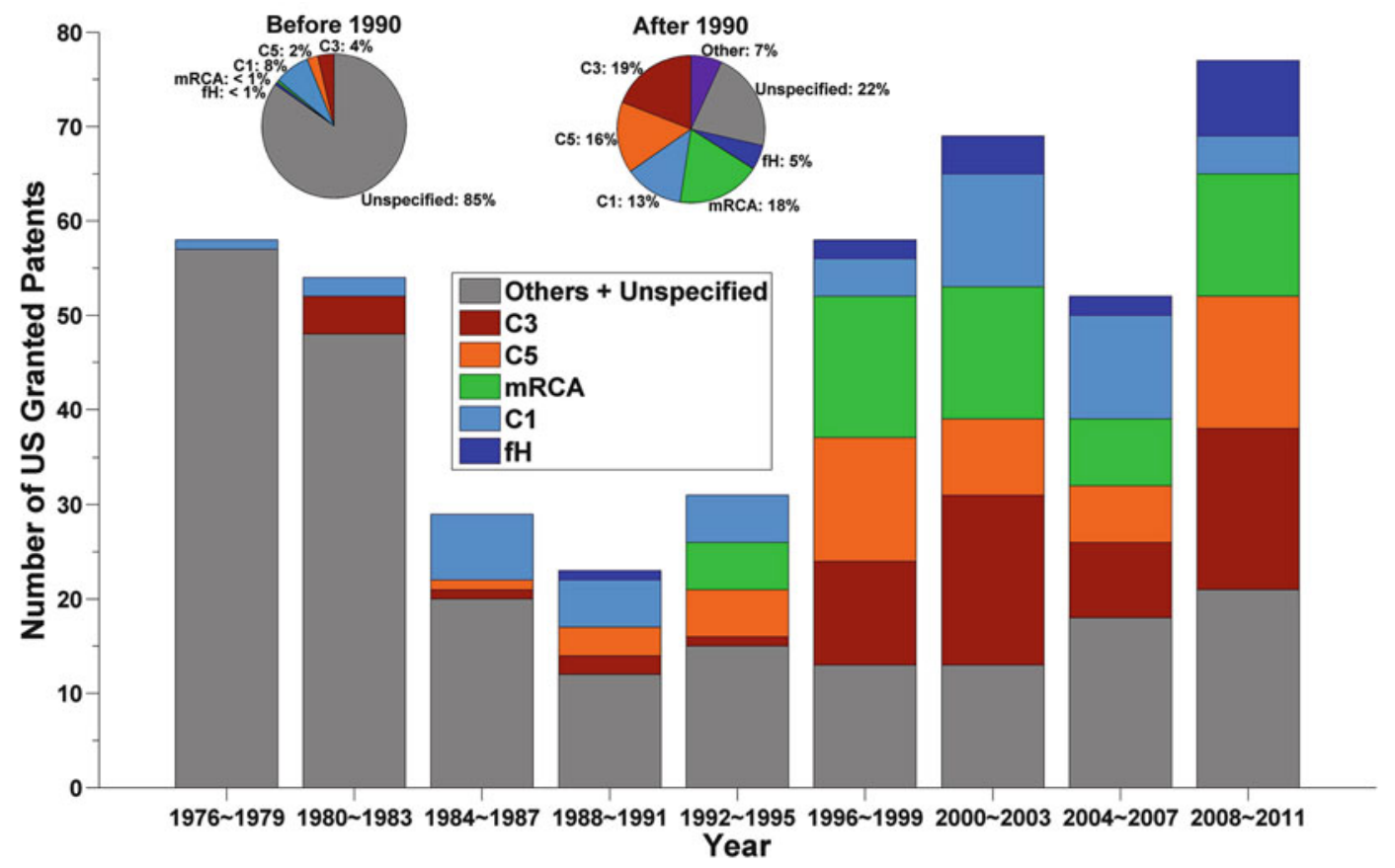

Fig. 21.3 Patent publication trends for the top five complement targets. The annual publication of US-granted patents related to one of the top five complement targets $(C 1, C 3, C 5, f H$, and $m R C A s)$ is plotted in 3-year periods. The distribution of patents for different targets before 1990 and from 1990 onward was also analyzed and charted, again showing specific distributions for the top five targets. "Other" refers to the remaining complement factors, such as C4, CR2, fB, $\mathrm{fD}, \mathrm{fI}$, and MBL-associated serine proteases (MASP), while "Unspecified" refers to publications that did not specify a specific complement target. Note that the "Other" category is not present in the pie chart showing patents prior to 1990 since these proteins were not the subject of any patents in that time period

$\mathrm{C} 5, \mathrm{fH}$, and mRCAs also grew rapidly during this time. It is not entirely surprising that $\mathrm{C} 3$ became such a strong focus for innovation. As the central component of the complement pathway, the regulation of $\mathrm{C} 3$ is an obvious choice for nearly complete control of complement activity. Accordingly, it is an attractive target for the development of complement inhibitors, such as the peptide inhibitor compstatin (Qu et al. 2011). In addition, the detection of C3 cleavage is a useful tool for determining the activation of the complement system, and thus innovations for assays to detect this event are highly applicable to complement research in general. Finally, C3 and/or its cleavage products have been linked to a variety of diseases and homeostatic and pathological conditions (Ricklin et al. 2010), further enhancing its appeal as a target for therapeutic regulation. Increased knowledge of the workings of the complement system since 1990 is also evident in the fact that most patents granted since that time have clearly described the mechanisms of their innovations. In fact, only $22 \%$ did not specify targets, and the ratio of patents with unspecified targets to total patents generally decreased yearly (Fig. 21.3, gray bar height compared to total bar height).

\subsubsection{Publication Trends Demonstrate Varied Applications of Complement Research and the Expansion of Inhibitor Forms}

There are many different complement-related innovations described in the patent literature, such as discovering inhibitors against complement activation, developing assays, detecting complement factors as biomarkers to diagnose diseases, and designing new procedures to produce and purify complement 

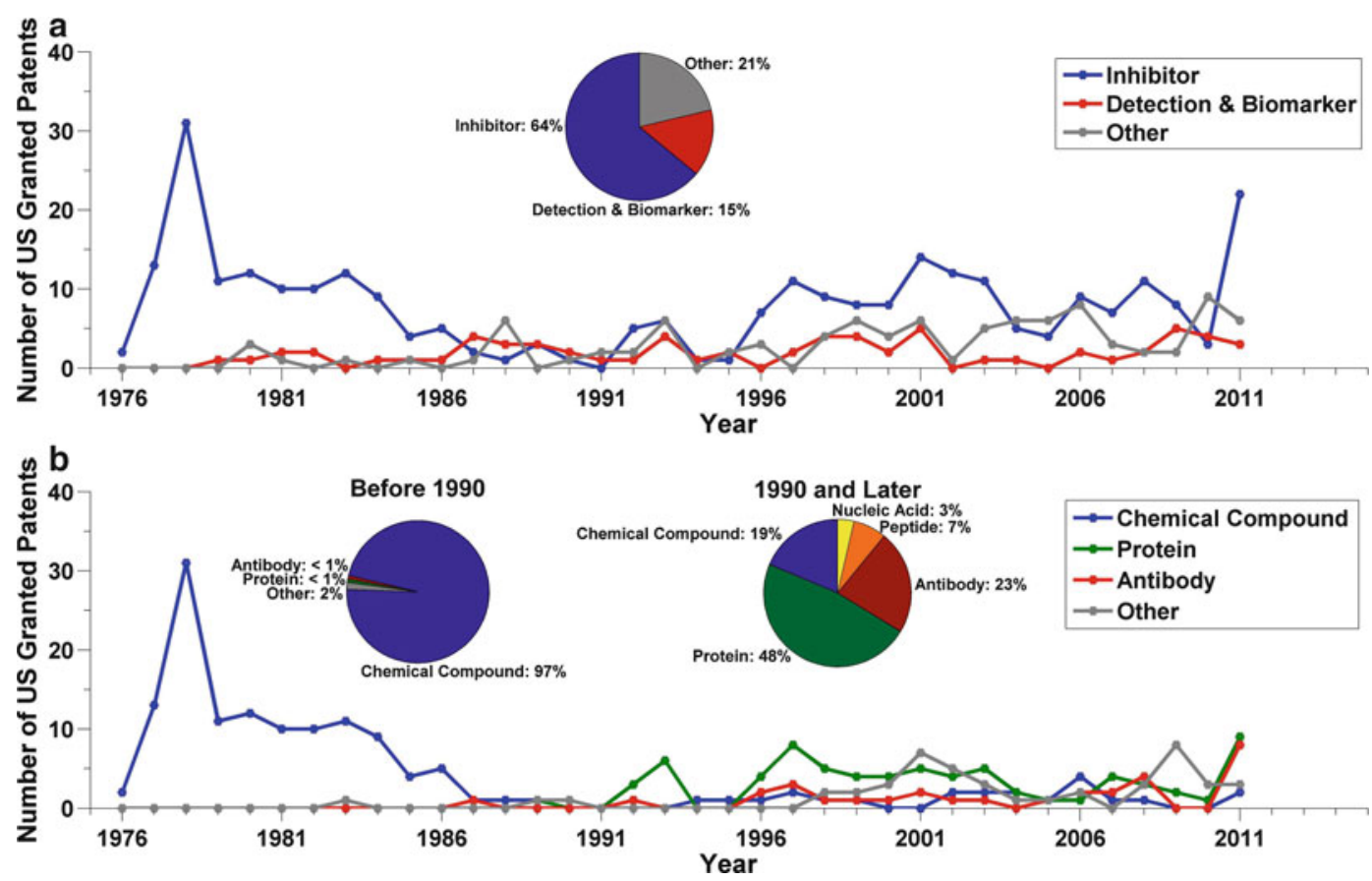

Fig. 21.4 Publication trends for complement-related patents based on applications or inhibitor forms. (a) The number of complement-related US-granted patents concerning inhibitor discovery, detection of complement components (including as biomarkers), or other applications (e.g., discovering novel complement-related proteins, producing animal models, describing crystal structures of complement factors, or developing absorbents to remove or immobilize complement components) is plotted to show annual trends. The overall distribution percentages are also charted. (b) The number of complement-related US-granted patents concerning inhibitors, as shown in (a), was further analyzed to determine what types of inhibitors were being proposed and developed, and the major forms are plotted to show annual trends. The data were also examined and charted to display distribution patterns before 1990 and from 1990 onward. "Other" in the graph refers to nucleic acids and peptides

components, among others. Of all these applications, the discovery of complement inhibitors has been the most prevalent, accounting for $64 \%$ of all publications (Fig. 21.4a, pie chart). Based on the yearly trends, the development of inhibitors was by far the major focus of early complement-related patents, and there has been another surge in interest over the past 10-15 years (Fig. 21.4a, graph). The granting of US patents for the detection of complement components, including biomarkers for disease diagnosis, and for other innovations has remained somewhat steady over the years, with both categories accounting for similar percentages of all publications (Fig. 21.4a, pie chart). A closer examination of the types of inhibitors being patented shows the overwhelming majority to be chemical compounds, proteins, and antibodies, which were the focus of at least $90 \%$ of all publications (Fig. 21.4b, pie charts). Before 1990, almost all complement inhibitors were chemical compounds, which were extensively synthesized by a broad range of pharmaceutical and chemical companies. Very frequently, the corresponding patents did not specify the exact target but instead described general complement inhibitors or inhibitory activity. For example, the American Cyanamid Company has patented more than 100 chemical compounds that can suppress complement activity in body fluids (Conrow and Bernstein 1978; Conrow et al. 1978; Lewis and Bernstein 1981). From 1990 onward, the interest in chemical compounds gradually shifted to antibodies and, largely, proteins. Only $19 \%$ of patents granted after 1989 claimed small synthetic compounds as complement inhibitors, while $71 \%$ of documents involved protein inhibitors and antibodies. Interestingly, a similar shift from small molecules toward biologics has been observed with FDA-approved drugs over the last 15 years (Mullard 2012). As revealed by 
yearly trends, the change in proportion for complement inhibitor-related patents seems to be due more to an overall decrease in the number of patents concerning inhibitors (Fig. 21.4a, graph), especially chemical compounds, rather than a large increase in the publication of other forms of inhibitors (Fig. 21.4b, graph). However, the increasing variety of and knowledge about compounds available for use as inhibitors in general since 1990 may explain the corresponding reemergence of patents related to complement inhibitors (Fig. 21.4a, graph), as the existence of new research tools likely spurred an interest in applying those tools to complement regulation.

Despite an overall strong interest in developing complement inhibitors, to date only two complement therapeutics have been approved by the FDA for use in humans (Ricklin and Lambris 2007). One is the purified glycoprotein C1 esterase inhibitor (C1-INH; Cinryze/ViroPharma, Cetor/Sanquin, Berinert/CSL Behring, Lev Pharma), and the other is a C5 antibody (Eculizumab; Soliris/Alexion Pharmaceuticals). Thus, it is not surprising that more and more attention has been paid to proteins and antibodies in recent years. Apart from chemical compounds, proteins, and antibodies, peptides and nucleic acids are the focus of the remaining $7 \%$ and $3 \%$ of all publications, respectively. Although they represent only a small fraction of all inhibitor-related publications, peptides and nucleic acids have been proposed as experimental therapeutics for several pathologies, and their potential future in disease treatment should not be neglected. For example, the peptidic C3 inhibitor compstatin and its analogs (Lambris and Sahu 2001; Lambris and Katragadda 2011) have been utilized and/or proposed for the treatment of eye disorders (Deschatelets et al. 2007), sepsis (Fung and Mollnes 2007), acute respiratory distress syndrome (ARDS) (Lambris and Ritis 2011), trauma (Francois et al. 2011a), Alzheimer's disease (Dinu 2007), pain (Woolf et al. 2006), and nerve regeneration (Baas and Ramaglia 2010), as well as other pathophysiological conditions.

\subsubsection{Emerging Disease Areas Drive New Patent Applications}

As mentioned, complement components are involved in many diseases, and they have been used as biomarkers for disease diagnosis, while complement inhibitors have been administered as therapeutics. Our analysis of US-granted patents has revealed eye disorders, transplants, cancer, and sepsis to be the major clinical conditions for which complement and its modulators have been utilized in a diagnostic or therapeutic sense (Fig. 21.5, pie chart).

In the case of eye disorders, about one-third of granted patents have claimed to use complement components to diagnose these diseases, while the other two-thirds focus on the application of complement inhibitors for the purpose of treatment. The three most common subjects of eye disorder-related patents have been $\mathrm{fH}, \mathrm{C} 3$, and $\mathrm{fB}$ (Fig. 21.5, graph). For example, polymorphisms in the $\mathrm{C} 3$, fH, and fB genes have been found to predict the occurrence of AMD (Thakkinstian et al. 2011; Zipfel et al. 2010) and thus have been used as diagnostic markers (Allikmets et al. 2011; Day et al. 2010). In other instances, a factor D antibody was patented as a treatment for complement-associated eye conditions such as AMD and choroidal neovascularization (CNV) (Hass et al. 2011). Eculizumab was patented by Alexion Pharmaceuticals, Inc. as a C5-specific antibody for therapeutic use in various complement-related diseases (Evans et al. 2002; Bell 2008; Wang and Matis 2007; Bell and Rother 2009; Rother et al. 2010) and is currently being evaluated for the treatment of AMD (Ricklin and Lambris 2007; Yehoshua et al. 2012). In addition, virus proteins such as smallpox inhibitor of complement enzymes (SPICE) and vaccinia virus complement control protein (VCP), both of which inhibit C3 activity, have been administered locally to the eye to treat disorders such as macular degeneration and choroidal neovascularization (Francois et al. 2011b).

In transplantation medicine, complement activity is known to critically contribute to inflammation and the accommodation or rejection of transplanted tissue (Asgari et al. 2010; Hughes and Cohney 2011). Complement inhibitors have been utilized in transplant recipients through various means to 


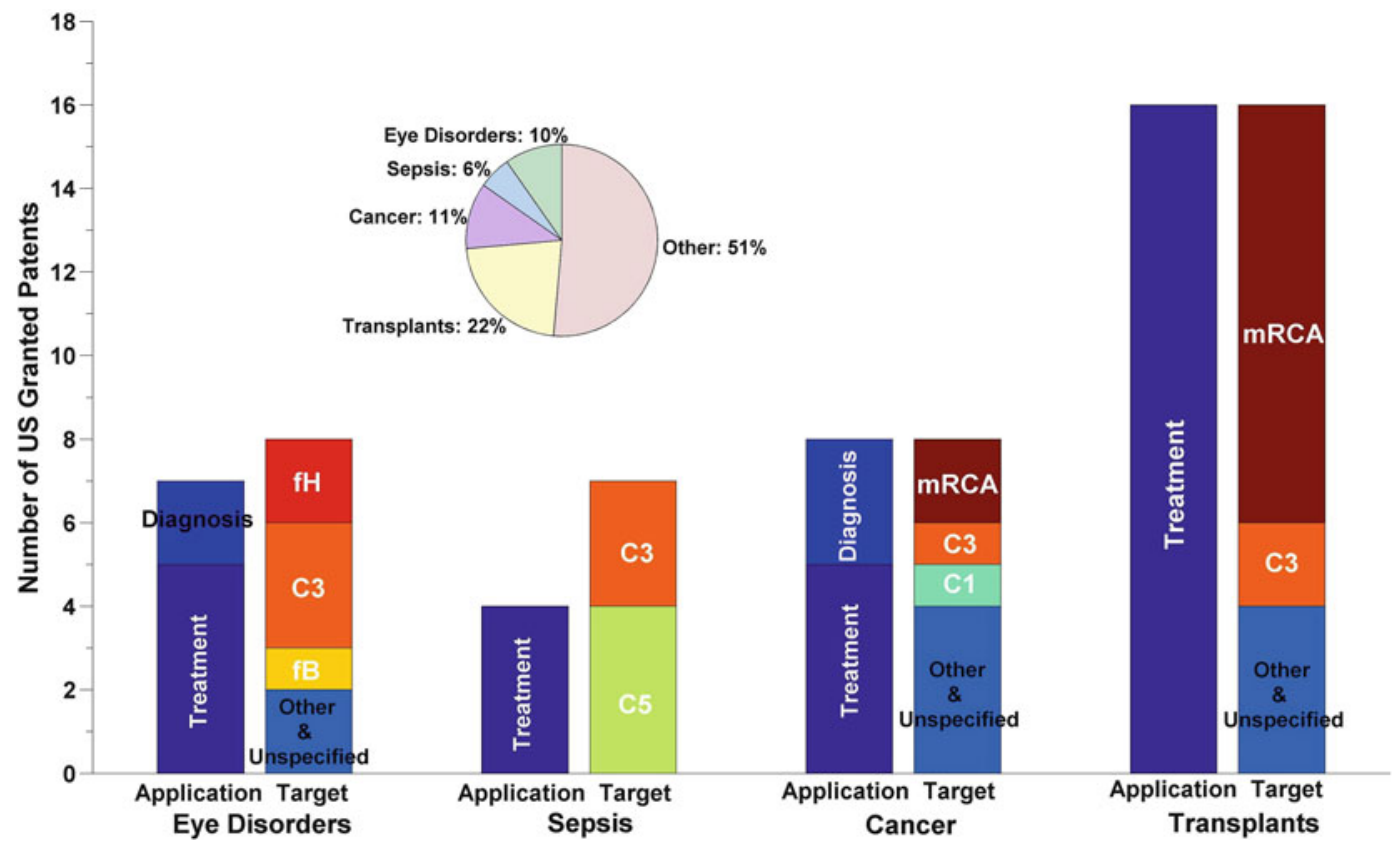

Fig. 21.5 Distribution of the application of complement and its modulators to various diseases and pathophysiological conditions. The chart displays the distribution percentages for US-granted patents that pertained to the use of complement and its modulators in the diagnosis and treatment of diseases and pathophysiological conditions, with "Other" referring to atherosclerosis, arthritis, SLE, Alzheimer's disease, spinal cord and neuronal injury, ischemia, infection, and other pathologies. The four main conditions described in these patents are shown in the graph: eye disorders (such as AMD), transplants, cancer, and sepsis. For each condition, the column on the left shows the number of patents related to treatment or diagnosis, and the column on the right indicates the top three complement components targeted, as well as others (in some cases, targets were not specified)

reduce the occurrence of adverse events against transplanted tissues, with mRCAs and C3 (or C3 convertases) being the most common points of intervention (Fig. 21.5, graph). Cells in transplanted tissues have been modified to express mRCAs, or organs have been perfused with membrane-targeted forms of recombinant RCAs in order to suppress complement activation and reduce the chances of complement attack on the tissue (Zhu 2007; Sims and Bothwell 1996; Smith et al. 2010, 2011). Transgenic animals expressing mRCAs have been produced for xenotransplantations (Diamond et al. 2000), and chimeric vaccinia virus proteins against $\mathrm{C} 3 \mathrm{~b}$ and $\mathrm{C} 4 \mathrm{~b}$ have been developed to prevent rejection by transplant recipients and improve the function of donor organs and tissues (Rosengard et al. 2000).

Complement and its modulators, especially mRCAs, $\mathrm{C} 3$, and $\mathrm{C} 1$, have been widely used in the diagnosis and treatment of cancer (Fig. 21.5, graph). One patented method for treating cancer has been the administration of an effective amount of a Coxsackie A-group virus, which recognizes and kills abnormal cells that express the mRCA DAF (Shafren 2008). C3b antibodies, alone or as conjugates with other antibodies, have been used to treat cancer through their binding to $\mathrm{C} 3 \mathrm{~b}$ on the surface of cancer cells (Taylor et al. 2003). Yet another patent describes analyzing patients' C1qA gene sequence as a means of predicting their response to CD20 antibody therapy (Racila and Weiner 2007).

Inhibitors against $\mathrm{C} 3$ and $\mathrm{C} 5$ have also been patented for the treatment of sepsis (Fig. 21.5, graph). An immunoadsorber with immobilized antibodies against C3a and/or C5a was developed for blood treatment in sepsis therapy (Heinrich et al. 2005) since excess activity of these anaphylatoxins can 
contribute to poor outcome in this disease (Bosmann and Ward 2012). Furthermore, complement and its modulators have been used in the diagnosis and treatment of arthritis, diabetes, SLE, ischemia, atherosclerosis, spinal cord and neuronal injury, and other diseases. For example, antibodies that inhibit the cleavage of C5 to C5a and C5b have been used to treat arthritis and prevent excessive downstream complement activation (Wang and Matis 2007). A C3 precursor biopolymer detected through the use of mass spectrometry has been utilized as a biomarker for type II diabetes (Jackowski and Marshall 2006). Finally, the levels of C4d and/or C3d on the surface of T lymphocytes, B lymphocytes, or monocytes in blood samples have been used to diagnose SLE (Ahearn et al. 2009). These are just a few examples of the many disease-related applications of complement that have been patented in the USA, yet they impressively illustrate the large diversity and creativity of complementrelated patents, as well as their potential impact on both biomedical research and public health.

\subsection{Conclusions and Outlook}

The complement system has long been known to be important for host defense against invading pathogens. This alone would make it an attractive target for potential therapeutic applications to enhance the immune response and fight infections. However, studies over the past few decades have revealed an increasing role for complement in a large variety of both pathological and homeostatic processes (Ricklin et al. 2010). In many instances, complement can be beneficial in one respect but becomes harmful to the host in another, namely, when its activity is not properly regulated. Thus, the appeal of targeting complement in an attempt to control its activation and effects has only grown as its many functions and cross-interactions with other biological systems have been increasingly revealed. Further increasing the attractiveness of targeting the complement system is the fact that this system consists of over 50 proteins, which for the most part act in a hierarchical pathway, with the later steps heavily dependent on actions that occur upstream. This situation presents a multitude of potential targets for managing complement activity. Even so, the regulation of complement has not been the only goal of the many innovations patented over the past four decades. Just as important has been the development of assays to detect complement activity, which have aided both experimental research and diagnostic practices. As a result of these new ideas and discoveries, interest in the complement system has continued to grow in the USA and around the world. In addition, the ability to manipulate the production and activity of many complement factors has led to the development of potentially life-saving diagnostic and therapeutic tools. Thus, it seems that the observed trend toward a constantly increasing wealth of complement-related innovations will continue well into the future.

\subsection{Disclosure}

J.D.L. holds several patents about the development and clinical application of complement inhibitors, including compstatin. He has previously served as a member on the Scientific Advisory Board of Potentia Pharmaceuticals and is the founder of Amyndas Biopharmaceutics, which perform clinical development of compstatin analogs for various indications.

Acknowledgments The authors wish to thank Deborah McClellan for editorial assistance. This work was supported by National Institutes of Health grants AI030040, AI068730, AI071028, AI072106, AI097805, GM097747, DE021685, and EY020633. 


\section{References}

Ahearn JM, Manzi SM, Liu C-C (2009) Diagnosing and monitoring inflammatory diseases by measuring complement components on white blood cells. US Patent 7,585,640

Allikmets RL, Hageman GS, Dean MC, Gold AM (2011) Variants in complement regulatory genes predict age-related macular degeneration. US Patent 8,012,683

Asgari E, Zhou W, Sacks S (2010) Complement in organ transplantation. Curr Opin Organ Transplant 15:486-491

Baas F, Ramaglia V (2010) Complement inhibition for improved nerve regeneration. US Application 20100143344

Bell L (2008) Methods for reducing morality associated with acute myocardial infarction. US Patent 7,361,339

Bell L, Rother RP (2009) Treatment of paroxysmal nocturnal hemoglobinuria patients by an inhibitor of Complement. US Application 20090220508

Bing DH (1983) Means and methods for purifying Clq, Clr and Cls. US Patent 4,374,061

Bosmann M, Ward PA (2012) Role of C3, C5 and anaphylatoxin receptors in acute lung injury and in sepsis. Adv Exp Med Biol 946:147-159

Conrow RB, Bernstein S (1978) 1,3,5- or 1,3,6-naphthalenetriyltris(sulfonylimino)aryl acids and salts. US Patent $4,087,613$

Conrow RB, Poletto JF, Bernstein S (1978) 5-Carboxy-phenylenebis(carbonylimino)benzene carboxylic and dicarboxylic acids and salts. US Patent 4,089,974

Day AJ, Clark SJ, Bishop PN, Sim RB, Blom AM, Heinegard D (2010) Screening assays for binding antagonists of the AMD-associated variant of factor $\mathrm{H}$ (H384). US Patent 7,829,301

Deschatelets P, Olson P, Francois C (2007) Compstatin and analogs thereof for eye disorders. US Application 20070238654

Diamond LE, Logan JS, Byrne GW, Sharma A (2000) Method of producing transgenic animals for xenotransplantation expressing both an enzyme masking or reducing the level of the gal epitope and a complement inhibitor. US Patent $6,166,288$

Dinu V (2007) Methods of preventing and treating Alzheimer's disease, age-related macular degeneration and other diseases involving extra-cellular debris through the inhibition of the complement system. US Application 20070196367

Evans MJ, Matis LA, Mueller EE, Nye SH, Rollins S, Rother RP, Springhorn JP, Squinto SP, Thomas TC, Wilkins JA (2002) C5-specific antibodies for the treatment of inflammatory diseases. US Patent 6,355,245

Francois C, Deschatelets P, Olson P (2011) Compositions and methods for treatment of trauma. US Application 20110092446

Francois C, Deschatelets P, Olson P (2011) Viral complement control proteins for eye disorders. US Patent 7,947,267

Fujii S, Yaegashi T, Nakayama T, Sakurai Y, Nunomura S, Okutome T (1986) Amidine compound, process for producing same and anti-complement agent comprising same. US Patent 4,570,006

Fujii S, Nakayama T, Nunomura S, Matsui R, Watanabe S-i, Sudo K, Okutome T, Kurumi M, Sakurai Y, Aoyama T (1987) Novel amidine compound. US Patent 4,634,783

Fung SC, Mollnes TE (2007) Methods and compositions for the prevention and treatment of sepsis. US Application 20070274989

Hallgren HR, Wide LE (1980) Method of indicating rheumatoid factors. US Patent 4,184,847

Hass P, Jianping Y, Katschke Jr K, Steffek M, Wiesmann C, Van Lookeren CM (2011) Prevention and treatment of complement-associated eye conditions. US Patent 8,007,791

Heinrich H-W, Hahn H-J, Meyer U, Kruschke P, Wagner H-J (2005) Immunoadsorber for use in sepsis therapy. US Patent 6,881,408

Holers VM (2003) The complement system as a therapeutic target in autoimmunity. Clin Immunol 107:140-151

Homann C, Varming K, Hogasen K, Mollnes TE, Graudal N, Thomsen AC, Garred P (1997) Acquired C3 deficiency in patients with alcoholic cirrhosis predisposes to infection and increased mortality. Gut 40:544-549

Hughes PD, Cohney SJ (2011) Modifiers of complement activation for prevention of antibody-mediated injury to allografts. Curr Opin Organ Transplant 16:425-433

Jackowski G, Marshall J (2006) Complement C3 precursor biopolymer markers predictive of type II diabetes. US Patent 7,097,989

Lambris JD, Katragadda M (2011) Potent compstatin analogs. US Patent 7,888,323

Lambris JD, Ritis K (2011) Method of treating acute respiratory distress syndrome. US Application 20110142837

Lambris JD, Sahu AK (2001) Peptides which inhibit complement activation. US Patent 6,319,897

Lewis AJ, Bernstein S (1981) C6-Modified cyclodextrin sulfate salts as complement inhibitors. US Patent 4,258,180

Markiewski MM, Lambris JD (2007) The role of complement in inflammatory diseases from behind the scenes into the spotlight. Am J Pathol 171:715-727

Markiewski MM, Lambris JD (2009) Unwelcome complement. Cancer Res 69:6367-6370 
Markiewski MM, DeAngelis RA, Lambris JD (2008a) Complexity of complement activation in sepsis. J Cell Mol Med $12: 2245-2254$

Markiewski MM, DeAngelis RA, Benencia F, Ricklin-Lichtsteiner SK, Koutoulaki A, Gerard C, Coukos G, Lambris JD (2008b) Modulation of the antitumor immune response by complement. Nat Immunol 9:1225-1235

Mullard A (2012) 2011 FDA drug approvals. Nat Rev Drug Discov 11:91-94

O'connell JP, Hoke RA, Mize PD (1987) Immunoassay using complement. US Patent 4,693,970

Pettigrew HD, Teuber SS, Gershwin ME (2009) Clinical significance of complement deficiencies. Ann N Y Acad Sci 1173:108-123

Pickering MC, Botto M, Taylor PR, Lachmann PJ, Walport MJ (2000) Systemic lupus erythematosus, complement deficiency, and apoptosis. Adv Immunol 76:227-324

Qu H, Magotti P, Ricklin D, Wu EL, Kourtzelis I, Wu YQ, Kaznessis YN, Lambris JD (2011) Novel analogues of the therapeutic complement inhibitor compstatin with significantly improved affinity and potency. Mol Immunol 48:481-489

Racila EV, Weiner GJ (2007) Assessing response to anti-CD20 therapy by genotyping C1q components. US Patent $7,723,036$

Ricklin D, Lambris JD (2007) Complement-targeted therapeutics. Nat Biotechnol 25:1265-1275

Ricklin D, Hajishengallis G, Yang K, Lambris JD (2010) Complement: a key system for immune surveillance and homeostasis. Nat Immunol 11:785-797

Rosengard AM, Ahearn Jr JM, Sanfilippo AP, Baldwin WM III (2000) Use of chimeric vaccinia virus complement control proteins to inhibit complement. US Patent 6,140,472

Rother RP, Wang H, Zhong Z (2010) Prolongation of survival of an allograft by inhibiting complement activity. US Application 20100135992

Shafren DR (2008) Methods for treating malignancies expressing ICAM-1 using coxsackie a group viruses. US Patent $7,361,354$

Sims PJ, Bothwell ALM (1996) Cells expressing high levels of CD59. US Patent 5,573,940

Skattum L, van Deuren M, van der Poll T, Truedsson L (2011) Complement deficiency states and associated infections. Mol Immunol 48:1643-1655

Smith RA, Dodd I, Mossakowkska DEI (2010) Conjugates of soluble peptidic compounds with membrane-binding agents. US Patent 7655617

Smith RAG, Pratt JR, Sacks SH (2011) Method of preparing an organ by perfusion. US Patent 7,888,318

Taguchi F, Mitsui I, Hara K, Hayashi M, Ezawa K, Fukunaga K, Kuranari J (1991) Test method involving substanceconjugated complement component C1q. US Patent 5,035,995

Taylor RP, Nardin A, Sutherland WM, Sokoloff MH, Chung L (2003) Methods for the prevention and treatment of cancer using anti-C3b(i) antibodies. US Patent 6,572,856

Thakkinstian A, McKay GJ, McEvoy M, Chakravarthy U, Chakrabarti S, Silvestri G, Kaur I, Li X, Attia J (2011) Systematic review and meta-analysis of the association between complement component 3 and age-related macular degeneration: a HuGE review and meta-analysis. Am J Epidemiol 173:1365-1379

Wang Y, Matis L (2007) Methods for the treatment of inflammatory joint disease. US Patent 7,279,158

Woolf C, Costigan M, Griffin R (2006) Methods for treatment of pain. US Application 20060241074

Yehoshua Z, Garcia Filho CAA, Gregori G, Li Y, Feuer WJ, Penha FM, Sadda SR, Zhang L, Zhang K, Rosenfeld PJ (2012) Systemic complement inhibition with eculizumab for the treatment of geographic atrophy in AMD patients: the complete study. In: The 2012 ARVO annual meeting

Zhu A (2007) Modified organs and cells for xenotransplantation. US Patent 7,166,278

Zipfel PF, Lauer N, Skerka C (2010) The role of complement in AMD. Adv Exp Med Biol 703:9-24 